\title{
Revascularización tardía en un paciente con infarto agudo del miocardio
}

Reporte de un caso y revisión de la literatura

Puentes Puentes Aurelio*, López Ramírez Cinthya Judith*, Hernández Godínez Efrén**, Ramírez Salazar Aristóteles**, Delgado Leal Luis***, Sandoval Rodríguez Eufracino**, Sánchez Hernández Ernesto****.

\section{Resumen}

- La principal causa de mortalidad en todo el mundo siguen siendo

- las enfermedades cardiovasculares, entre las que destacan por su

- frecuencia los síndromes coronarios agudos (SCA) contribuyen sus-

- tancialmente, a corto y largo plazo. Por tal motivo, las decisiones mé-

- dicas que se toman en cada caso clínico, influyen notablemente en

- la función cardiaca del paciente y esto determina tanto la calidad de

- vida como la sobrevida en todos los enfermos. Presentamos un caso

- y la justificación que existe en la literatura médica de cómo hacer

- una buena práctica médica, orientada a mejor pronóstico. LUXMÉDICA

- AÑO8, NÚMERO24, MAYO-AGOSTO 2013, PP 37-42

-

Palabras clave: TIMI, GRACE, SYNTAX, coronariografía, angioplastía.

\section{Abstract}

The main cause of mortality in the world are still cardiovascular diseases, including acute coronary syndromes(ACS) that substantially contribute to short and long term by its frequency. For this reason medical decisions that are taken in each clinical case, significantly influencing the cardiac function of the patient and this determines both quality of life and survival in all patients. We present a case and justify that exists in the medical literature of how to make a good medical practice, aimed at better prognosis. LUX MÉDICA AÑ0 8, NÚMERO 24, MAYO-AGOSTO 2013, PP 37-42

key words: TIMI, GRACE, SYNTAX, coronariography, angioplasty.

\section{Introducción}

Se conoce por estudios reportados por varios autores, que la incidencia de infarto agudo del miocardio está alrededor de 1-2 casos de cada 500 habitantes por año. Dentro de estas cifras se incluyen el síndrome coronario agudo (SCA) con elevación del ST o sin elevación del segmento ST. A pesar de los importantes avances diagnósticos y terapéuticos que se han suscitado en las últimas cuatro décadas, el infarto agudo del miocardio con elevación del segmen-

* Médico Residente de segundo año de Cardiologia del Centenario Hospital Miguel Hidalgo.

** Médico Residente de tercer año de Cardiología del Centenario Hospital Miguel Hidalgo.

*** Médico adscrito al servicio de Hemodinamia del Centenario Hospital Miguel Hidalgo.

**** Médico adscrito al servicio de Medicina Nuclear del Centenario Hospital Miguel Hidalgo.

Fecha de recibido: 11 de abril 2013

Fecha de aceptación: 23 de julio 2013

Correspondencia: Dr Aurelio Puentes Puentes. Residente de segundo año de Cardiología del Centenario Hospital Miguel Hidalgo. Calle Galeana Sur No. 465 Colonia Obraje CP 20230, Aguascalientes, Ags. México. Teléfono 01(449) 9946720, Correo electrónico aurelio_p83@hotmail.com. 
to ST continua siendo un problema importante de salud pública en todo el mundo. Solo en los Estados Unidos de América cerca de un millón de personas presentan este tipo de infarto cada año y estiman que unas 300 mil personas más, fallecen antes de llegar a ser hospitalizados. ${ }^{1,2}$. De tal manera que el manejo oportuno en estos pacientes es de vital importancia para su pronóstico a corto y mediano plazo. Presentamos un caso de un síndrome coronario agudo que llega fuera de ventana terapéutica (12 hs), para manejo trombolítico o de angioplastía coronaria primaria. Se revisó la literatura que justifica el manejo realizado. ${ }^{3}$

\section{Presentación del caso clínico}

Se trata de paciente del sexo masculino, de 55 años, comerciante, que se le identifica como fumador como único factor de riesgo, con un Índice tabáquico pulmonar de 20 paquetes/año. El 23 de febrero presenta dolor torácico de tipo opresivo con intensidad 8/10, con duración de 30 minutos y diaforesis. El 25 de febrero se tomó un electrocardiograma (ECG) (fig. 1) y se diagnosticó infarto agudo del miocardio con elevación de segmento ST (IAMCEST), fuera de ventana para trombolisis o angioplastía primaria. Con una tensión arterial de $95 / 50 \mathrm{mmHg}$, frecuencia cardiaca (FC) 100 lpm, frecuencia respiratoria $20 \mathrm{rpm}$, índice de masa corporal de $29 \mathrm{~kg} / \mathrm{m}^{2}$. En precordio presenta impulso apical en 5 Ell en LMC, normo dinámico, con un cuarto ruido intermitente, sin soplos ni frote pericárdico, con estertores pulmonares. Los resultados de laboratorio del paciente mostraron: glucemia $118 \mathrm{mg} / \mathrm{dl}$, creatinina de $0.79 \mathrm{mg} / \mathrm{dl}$, depuración de creatinina por fórmula de Crockroft de $112 \mathrm{ml} / \mathrm{min}$, sodio de $135 \mathrm{mEq}$ y potasio de $4.5 \mathrm{mEq}$. Perfil de lípidos al ingreso con colesterol total de $287 \mathrm{mg}$, triglicéridos de $122 \mathrm{mg}$, HDL de 24.6 y LDL de $238 \mathrm{mg} / \mathrm{dl}$. El ECG mostró un ritmo sinusal con FC de 85 lpm y lesión subepicárdica anterior e isquemia subepicárdica lateral alta, necrosis anterior (QS y elevación del ST en V1-2, T negativas en $\vee 4-6)$ (fig. 1a) La radiografía de tórax mostró datos de hipertensión venocapilar pulmonar (fig. 1b).

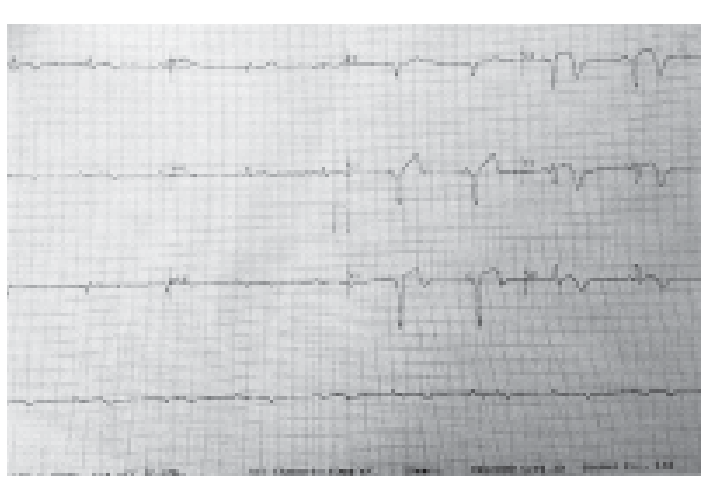

Fig. la ECG al ingreso

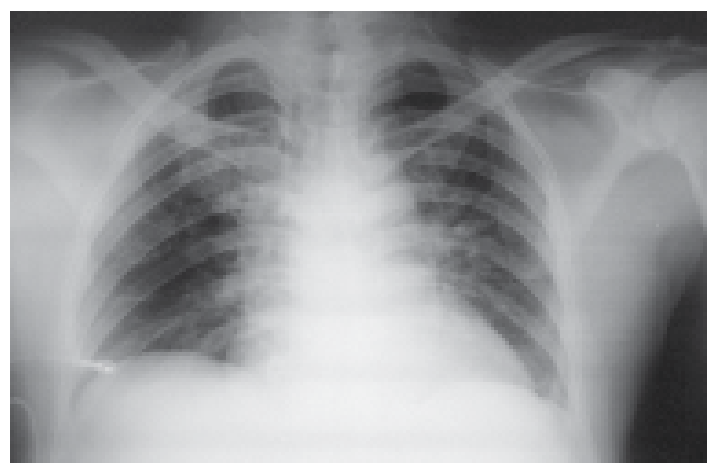

Fig. Ib Radiografía Tórax 
El diagnóstico fue de infarto agudo al miocardio con elevación del segmento ST(IAMCESST) Killip-Kimball II, escala TIMI de 9 puntos (mortalidad a los 30 días de $27 \%$ ) y GRACE de 178 puntos (mortalidad de $8 \%$ intrahospitalaria) y GRACE de 132 puntos a 6 meses (mortalidad del $12 \%$ ) sin reperfusión mecánica ni farmacológica, con una fracción de eyección del ventrículo izquierdo de $46 \%$ y alteraciones en la movilidad segmentaria con acinesia septo-apical y anterior e hipocinesia moderada anterior por ecocardiografia realizada el 26/02/13.
El 27/02/13 se decidió estratificación invasiva (coronariografía) por riesgo alto en escalas de TIMI (9 puntos), GRACE (178 puntos), mala función ventricular (FEVI 46\%) y troponinas muy elevadas. Se encontró enfermedad coronaria trivascular con una valoración por la escala de SYNTAX de 19 puntos $^{4,5}$ (fig. 4) por lo que se realizó angioplastia coronaria de la descendente anterior (vaso de $3 \mathrm{~mm}$ y lesión suboclusiva concéntrica del $90 \%$ tipo C con diámetro de $26 \mathrm{~mm}$ ), de la marginal obtusa de la circunfleja, y de la coronaria derecha, con éxito técnico y éxito primario.

Figura 4a, $4 b$ y 4c Coronariografía y figura 4d, 4e y 4f Angioplastía coronaria

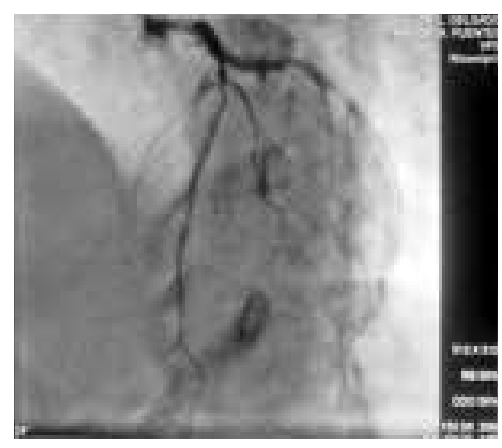

a) DA proximal $90 \%$

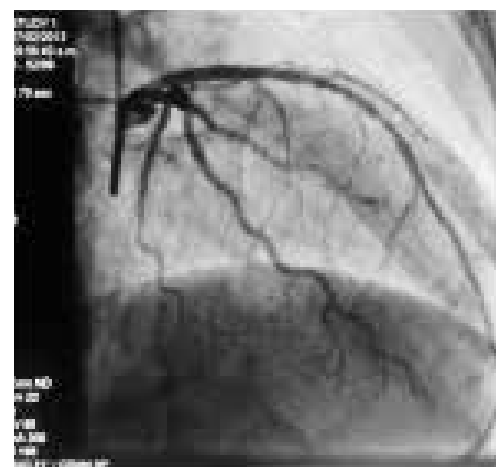

d) Stent Endeavor Resolute $3 \times 30 \mathrm{~mm}$

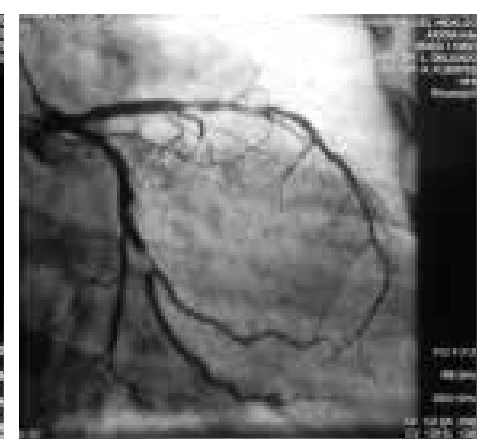

b) $10 M 76 \&$

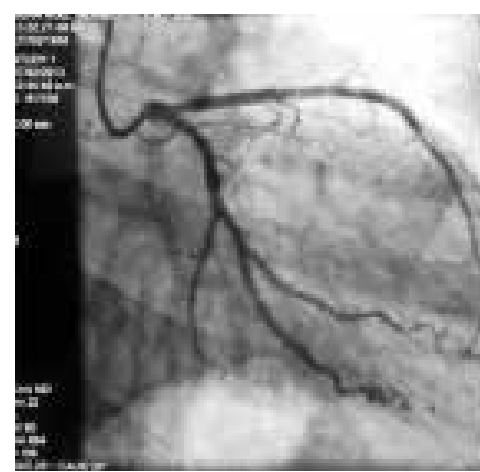

e) Stent Driver Rx $3.0 \times 18 \mathrm{~mm}$

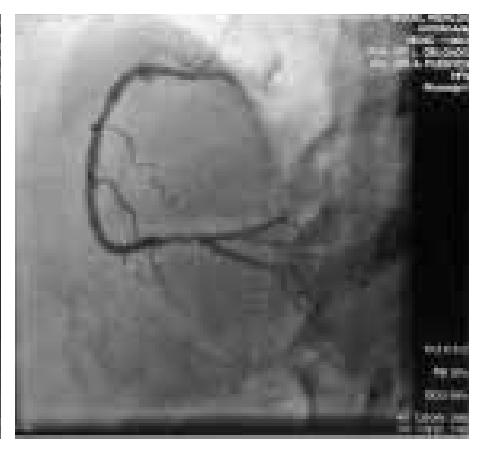

c) CD distal $75 \%$

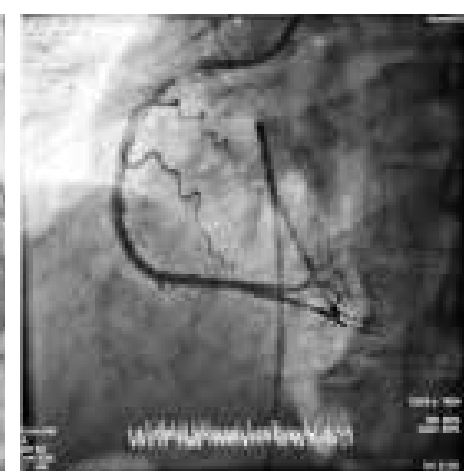

f) Stent Driver Rx $3.0 \times 15 \mathrm{~mm}$

\section{Discusión y revisión}

Este es un caso muy común en la práctica hospitalaria, ya que un alto porcentaje de enfermos llega después de 12 horas de haber iniciado con el dolor precordial, es decir, fuera de la ventana terapéutica estipulada. Los resultados de las variables nos hicieron catalogar al paciente como de riesgo alto, mediante la valoración con escalas deTIMI y GRACE, además de resultar 
con troponinas muy elevadas y baja medición de función ventricular, por lo que se justificó la estrategia invasiva en este caso en particular. ${ }^{6,7}$

Para valorar el paciente con infarto agudo del miocardio con elevación del segmento ST ocasionado por un síndrome coronario agudo, existen varias escalas de riesgo, como son TIMI (valora las variables: edad >75 años: 3 puntos, 65-74 años: 2 puntos, diabetes mellitus tipo 2/hipertensión arterial sistémica: 1 punto, presión arterial sistólica $<100 \mathrm{mmHg}$ : 3 puntos, frecuencia cardiaca $>100 \mathrm{lpm}: 2$ puntos, KK II-IV: 2puntos, peso <67 kg: 1 punto, localización anterior o BRIHH: 1 punto y tiempo de tratamiento $>4$ hrs: 1 punto), CADILLAC, PAMI y GRACE (valora las variables: edad, IC, infarto previo, frecuencia cardiaca, presión arterial sistólica, descenso del ST, creatinina y biomarcadores iniciales estratificándose con riesgo bajo [98-108 puntos], riesgo intermedio [109-140 puntos] y alto [ $>140$ puntos]), todos considerados como herramientas excelentes para la estratificación de riesgo y valorar el pronóstico de mortalidad en los pacientes. Sin duda, estas herramientas nos orientan a tomar mejores decisiones con evidencias. ${ }^{8}$ Las escalas TIMI, CADILLAC y GRACE poseen una mayor capacidad predictiva para la mortalidad a 30 y 365 días en comparación al estudio PAMI. ${ }^{9}$ En nuestro hospital se utilizan de forma rutinaria las escalas TIMI y GRACE.

Las guías establecen primordial la terapia de reperfusión del tejido miocárdico cuando se encuentra dentro de las primeras doce horas desde el inicio de los síntomas y con elevación persistente del ST o BRI$\mathrm{HH}$ nuevo, preferentemente para $\mathrm{PCl}$ primaria a los 90 minutos del primer contacto médico y aceptable a los 120 minutos. Si este objetivo no se puede alcanzar, lo recomendable es la fibrinólisis y después de una fibrinólisis efectiva se recomienda la angiografía a las 3-24 hrs. ${ }^{10}$ Los pacientes que se presentan entre las 12 y 24 horas y posiblemente hasta las 60 hors tras la aparición de los síntomas, incluso cuando no tienen dolor y la función hemodinámica es estable, pueden beneficiarse de una coronariografía inmediata y si fuera preciso de angioplastía coronaria transluminal percutánea (ACTP).

Por lo tanto la ACTP es el tratamiento de elección en los pacientes que se presentan idealmente a los 90 minutos y aceptable hasta los 120 minutos en pacientes con IAMCESST, es de importancia recordar que en esta población se ha encontrado un $41-67 \%$ de enfermedad arterial coronaria multivaso, cifra que depende de las características de los pacientes (por ejemplo: la edad), de los cuales solo $10 \%$ de los pacientes que son llevados a ACTP tiene indicación para ACTP de las arterias no responsables del infarto, de acuerdo a los resultados de un estudio que llevó un seguimiento de 3 años. La importancia de la enfermedad multivaso radica en su asociación con un pronóstico malo en pacientes con IAMCEST en fase aguda tal como se describe enseguida: $:^{11,12}$

a) Inestabilidad de la placa no solo se limita a la arteria responsable del infarto.

b) La revascularización completa se asocia con mejor pronóstico a largo plazo.

c) La ACTP está justificada en pacientes con IMACEST en fase aguda (inestabilidad hemodinámica, Insuficiencia cardiaca (IC), arritmias y paro cardiaco).

d) Cursa con un estado protrombótico e inflamatorio alto.

e) Mayor grado de espasmo, por lo que se puede sobreestimar la estenosis de arterias no relacionadas con el infarto del miocardio.

f) Mayor utilización de contraste y mayor riesgo de nefropatía por contraste.

Los pacientes sin dolor torácico o isquemia inducible que se presentan a los 3-28 días con una oclusión coronaria persistente no obtienen este beneficio de la ACTP ${ }^{13}$, sin embargo se considera que los pacientes que 
se presentan dias después del episodio agudo con un infarto del miocardio, solo debe considerarse la revascularización cuando la arteria esté ocluida y condicione angina recurrente o isquemia residual documentada y viabilidad tisular por un método de imagen no invasiva en un territorio miocárdico extenso. El caso que mostramos da una idea de cómo actuar en un paciente que de inicio pareciera estable, pero con evidencias de riesgo elevado como se constató conforme se integró totalmente el caso.

El score SYNTAX es un modelo estadístico aplicado a pacientes con lesión de tronco o con 2-3 arterias epicárdicas coronarias obstruidas, cuyo diseño se basó en otras escalas y clasificaciones como: el estudio ARTS, el score Leaman, el sistema de clasificación de la ACC/AHA, el sistema de clasificación de oclusión total, la clasificación de Dukes y de ICPS para lesiones bifurcadas y el consenso de expertos, el SYNTAX se enfoca en predecir un pronóstico basándose en la anatomía coronaria y su complejidad a partir de las siguientes variables: a) dominancia, b) número de lesiones, c) oclusión total, d) trifurcación, e) bifurcación, f) estenosis aorto ostial, g) tortuosidad severa, h) longitud $>20 \mathrm{~mm}$, i) calcificación, j) trombo y k) enfermedad de vaso difuso/vaso pequeño, ubicando a los pacientes con un puntaje mayor de 22 como óptimos para revascularización por cirugía y menor a este puntaje como candidatos a intervencionismo percutáneo. ${ }^{14}$ El estudio ACUITY que incluyó 13819 pacientes con SCA de alto riesgo comparó ambas estrategias de revascularización coronaria, intervencionismo percutáneo (PCI) vs cirugía de revascularización coronaria (CRC) en 5627 pacientes que tenían enfermedad multivaso de los cuales 4412 fueron llevados a PCI y 1215 a CRC concluyendo que la sobrevida era similar y solo aumento el riesgo de complicaciones en el grupo de $\mathrm{PCI}$ (EVC, IRA, sangrado y hospitalización) ${ }^{15}$. En otro estudio que incluyó 5775 pacientes diabéticos y no diabéticos no se encontró diferencias significativas en los métodos de revascularización coronario quirúrgico o por $\mathrm{PCl}$, solo se asoció la $\mathrm{PCl}$ a mayor riesgo de nueva revascularización . ${ }^{16}$

\section{Conclusiones}

El caso que presentamos denota un enfermo con estratificación no invasiva que lo cataloga como un paciente de alto riesgo en quien está indicada la intervención invasiva por los beneficios que se obtiene tanto en el pronóstico e incremento de la función ventricular, como objetivo final del tratamiento.

\section{Bibliografía}

1. American heart association. Heart and stroke statistic abrev 2001. Dallas TX. American Heart Association.

2. British Heart Foundation. Coronary heart disease statistic. 2004. London: Brithis Heart Fundation.

3. Lucas JW, Schiller JS. Sumary Healt Statistic for USA. Adults: National Heart Intervien Survey, Vital Healt 10.2004;218:1-134.

4. Grupo de trabajo para el manejo del infarto agudo de miocardio con elevación del segmento ST de la SEC. Guía de la práctica clínica de la ESC para el manejo del infarto agudo de miocardio en pacien- tes con elevación del segmento ST. Rev Esp Cardiol. 2013;(1):53.e1-e46.

5. Georgios S., et al. The SYNTAX Score: an angiographic tool grading the complexity of coronary artery disease. Eurolnterv.2005;1:219-227.

6. Sergio Raposeiras R, Emad Abu S, Pilar Cabanas G, Walking Beyond the GRACE (Global Registry of Acute Coronary Events) Model in the Death Risk Stratification During Hospitalization in Patients With Acute Coronary Syndrome. JACC: Card Interv 2012: 5(11);1117-25.

7. Morrow DA, Antman EM, Charlesworth A et al. TIMI risk score for ST-elevation myocardial infarction: 
A convenient, bedside, clinical score for risk assessement at presentation: An intravenosus nPA for treatment of infarvting myocardium early II trial substudy. Circulation 2000; 102; 203-7.

8. Méndez Eirín E, Flores Ríos X, García López F. Comparación del valor predictivo pronosticó de los scores TIMI, PAMI, CADILLAC y GRACE en el SCACEST sometido a ICP primario o de rescate. Rev Esp Cardiol. 2001;65(3):227-233.

9. Baptista SB, Farto e Abreu P, Lourerio JR, Thomas B, Nedio $M$, Gago S, Ferreira R. PAMI risk score for mortality prediction in acute myocardial infarction treated with primary angioplasty. Rev Port Cardiol, 2004: 23(5):683-93.

10. Grupo de trabajo de Revascularización Miocárdica de la SEC y de la Asociación Europea de Cirugía Cardiotorácica. Guía de práctica clínica sobre revascularización miocárdica. Rev Esp Cardiol. 2010;63(12):1485 e1-e76.

11. Thygesen $K$, Alpert JS, Jaffe AS, Simoons ML, Chatmain BR. Third Universal Definition of Myocardial Infarction. Journal of the Amer Coll Card. 2012;60:1-18.

12. Petr Widimsky and David R. Holmes Jr. How to treat patients whit ST-elevation acute myocardial infarction and multi-vassel disease. Europ Heart Journal 2010.

13. Baz JA, Iñiguez Romo A, García Fernández E, Serra Peñaranda A, Macaya Miguel C. Tratamiento de reperfusión en el infarto agudo de miocardio con elevación del segmento ST. Rev Esp Cardiol Supl. 2010;10:12C-20C.

14. Leaman MD, Brower RW, Meester GT, Serruys $P$, van den Brand $M$. Coronary artery atherosclerosis: severety of the disease, severety of angina pectoris and compromised left ventricular function. Circulation. 1981;63(2):285-99.

15. Ben-Gal Y, Moses JW, Mehran R and et al. Surgical versus Percutaneous Revascularization for Multivessel Disease in Patients with Acute Coronary Syndromes. JACC: Cardiovascular Interventionts2010;3(10): 1059-1067.

16. Park DW and et al. Long-Term Outcome of stents Versus Bypass Surgery in Diabetic and Nodiabetic Patients Whit Multivessel or Left Main Coronary Artery Disease. Circ Cardiovasc Interv 2012;5:467475. 